Canadian

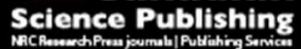

Canadian Journal of Forest Research Revue canadienne de recherche forestière

\title{
Comparison of methods for spatial interpolation of fire weather in Alberta, Canada
}

\begin{tabular}{|r|l|}
\hline Journal: & Canadian Journal of Forest Research \\
\hline Manuscript ID & cjfr-2017-0101.R2 \\
\hline Manuscript Type: & Article \\
\hline Date Submitted by the Author: & 03-Oct-2017 \\
\hline Complete List of Authors: & $\begin{array}{l}\text { Jain, Piyush; University of Alberta, Renewable Resources } \\
\text { Flannigan, Mike; University of Alberta, Renewable Resources }\end{array}$ \\
\hline Keyword: & $\begin{array}{l}\text { interpolation, Kriging, Fire Weather Index, Inverse distance weighting, } \\
\text { Splines }\end{array}$ \\
\hline $\begin{array}{r}\text { Is the invited manuscript for } \\
\text { consideration in a Special } \\
\text { Issue? : }\end{array}$ & N/A \\
\hline
\end{tabular}


3

4

$$
\text { P. Jain }{ }^{\mathrm{A}} \text { and M.D. Flannigan }{ }^{\mathrm{A}}
$$




\section{$7 \quad$ Abstract}

8 Spatial interpolation of fire weather variables from station data allow fire danger indices to be

9 mapped continuously across the landscape. This information is crucial to fire management

10 agencies, particularly in areas where weather data are sparse. We compare the performance of

11 several standard interpolation methods (inverse distance weighting, spline and geostatistical

12 interpolation methods) for estimating output from the Canadian Fire Weather Index (FWI)

13 System at unmonitored locations. We find geostatistical methods (Kriging) generally outperform

14 the other methods, particularly when elevation is used as a covariate. We also find that

15 interpolation of the input meteorological variables as well as the previous day's moisture codes

16 to unmonitored locations followed by calculation of the FWI output variables is preferable to

17 first calculating the FWI output variables and then interpolating, in contrast to previous studies.

18 Alternatively, when the previous day's moisture codes are estimated from interpolated weather,

19 rather than directly interpolated, errors can accumulate and become large. This effect is

20 particularly evident for the duff moisture code and drought moisture code, due to their significant

21 autocorrelation.

23 Keywords: Interpolation, Kriging, Fire Weather Index, Inverse distance weighting

24 Splines 


\section{Introduction}

Wildfire is not only a natural disturbance on ecosystems but is also a major component of

27 forest management, particularly in areas where fire coexists with human activity and values. In

28 addition, anthropogenic climate change is expected to increase both fire frequency and severity

29 in the coming century (Wang et al. 2015). On a daily timescale wildfire potential has a strong

30 dependence on antecedent and current weather conditions. For example, in Canada, 97\% of the

31 area burned by wildland fire is the result of just 3\% of fire ignitions (Stocks et al. 2002). These

32 large fires occur on days with extreme fire weather (Flannigan and Harrington 1988); that is,

33 days with a combination of meteorological conditions conducive to a highly elevated wildfire

34 risk. Various fire weather indices are used by many countries to inform fire management

35 agencies and the public about potential fire danger due to meteorological conditions. This

36 information is used to employ preventative measures (eg. prescribed burning), for the allocation

37 of resources for fire control and suppression, or for emergency response (eg. evacuations). These

38 fire management goals are facilitated by the accurate spatial mapping of fire danger on the

39 landscape.

Widely used fire danger indices include the US National Fire Danger Ratings System

41 (NFDRS) (Deeming et al. 1977), the Australian McArthur Forest Fire Danger Index (McArthur

42 1967), the Haines Index (Haines, 1988), and the Fire Weather Index (FWI) System, which is part

43 of the Canadian Forest Fire Danger Rating System (CFFDRS) (Van Wagner 1987). The FWI

44 system is used extensively by provinces and territories in Canada to monitor wildfire risk due to

45 weather conditions. The input to the FWI system consists of daily noon observations of

46 temperature, relative humidity, wind speed and 24 hour accumulated precipitation. The system

47 works by calculating three intermediate moisture codes which represent the moisture content of

48 three ground layers. These are the Fine Fuel Moisture Code (FFMC), the Duff Moisture Code 
49 (DMC) and the Drought Code (DC). Subsequently two indices, the Build-up index (BUI) and

50 Initial Spread Index (ISI) are calculated, which are combined to give an overall Fire Weather

51 Index (FWI) or its transformed equivalent the Daily Severity Rating (DSR). The variable

52 dependence of the FWI system is illustrated in Figure 1. An important feature of the system is

53 that the FWI System outputs for the current day depend not only on the current day's weather but

54 the previous day's moisture codes. For this reason, the system is essentially a bookkeeping

55 system for fuel moisture.

56 Most provincial fire management agencies in Canada deploy their own weather station

57 networks specifically to monitor fire weather during the fire season. The number and location of

58 weather stations is constrained by cost, location of values and convenience. The mapping of fire

59 danger on the landscape therefore usually requires spatial interpolation of meteorological

60 variables or fire weather indices onto a grid. Spatial interpolation refers to any method that

61 estimates a value of a variable (or variables) at an unobserved location (predictand) using data at

62 observed locations (predictors). The accuracy of interpolated weather in general depends on the

63 following: i) the spatio-temporal characteristics of meteorological variables ii) measurement

64 error, iii) station network density, iv) station network distribution, iv) topography or other local

65 climate factors; and vi) chosen interpolation method. Based on an early study using data from

66 New Brunswick, FWI values are expected to be representative of conditions within a $40 \mathrm{~km}$

67 radius surrounding a weather station, becoming unreliable beyond a radius of $160 \mathrm{~km}$ (Williams

68 1963). These values have subsequently been used as a guide for Canadian fire management

69 agencies (Lawson and Armitage 2008). In the 1990s the spatial Fire Management System

70 (sFMS) was developed, adding GIS capabilities to the CFFDRS and providing a computerized

71 forest fire management system to be used operationally (Lee et al. 2002). Part of these added

72 capabilities included the ability to map fire danger using spatial interpolation, with the inverse 
73 distance weighting scheme being used by default (Englefield et al. 2000). Currently the sFMS

74 interpolates fire weather by interpolating the meteorological variables to grid points and then

75 calculating the FWI components. The previous day's moisture codes are taken from the previous

76 day's calculated codes on the grid, unless no value is available (as occurs early in the fire

77 season), in which case the codes are interpolated from available weather stations (P. Englefield

78 (personal communication, 2017)).

There are a few studies specifically examining interpolation of fire danger rating indices.

80 Flannigan and Wotton (1989) previously compared interpolation methods for the Canadian FWI

81 system in the North Central Region of Ontario. They tested a second order polynomial fit, cubic

82 spline (thin plate spline) and weighted interpolation (inverse distance weighting) for

83 interpolation of the FWI. Their results suggest the smoothed cubic spline gives the most

84 consistent results. Flannigan et al. (1998) investigated whether interpolation of FWI could be

85 improved by using precipitation estimates from radar. Tait and Zheng (2005) also compared a

86 number of interpolation schemes for the FWI over New Zealand and found the thin plate

87 smoothed spline performed best. Significantly both of these studies concluded that direct

88 interpolation of the FWI was preferable to interpolation of the underlying meteorological

89 variables followed by calculation of the FWI. Sanabria et al. (2013) examined the spatial

90 interpolation of the Australian fire danger rating system, the McArthur Forest Fire Danger Index

91 (FFDI). They achieved the best results with an algorithm that combined random forest and

92 inverse distance weighting interpolation. This study focused specifically on return intervals of

93 extreme values of the FFDI. More generally, the interpolation of meteorological variables has

94 been treated extensively in the literature and we refer the interested reader to Li and Heap (2014)

95 who review a number of commonly applied interpolation methods. 
More recently, a number of alternatives to spatial interpolation have been suggested for

97 real-time monitoring or forecasting of fire danger. Satellite remote sensing has been investigated

98 for real-time monitoring of live fuel moisture (Leblon 2001). Although satellite remote sensing

99 of land surface temperature (Li et al. 2013) and precipitation (Stephens and Kummerow 2007) is

100 possible, the technology has not yet advanced sufficiently for determination of land surface wind

101 speed and relative humidity, which are also required meteorological inputs of the FWI System.

102 The use of gridded products such as the Canadian Precipitation Analysis (CaPA) (Mahfouf et al.

103 2007), which blends gauge data, numerical weather forecasts and radar estimates, has also been

104 investigated for improving precipitation and fuel moisture estimates using the FWI System

105 (Hanes et al., in press). On the other hand, there are (early warning) fire danger forecasting

106 systems based on numerical weather models at both the continental (San-Miguel-Ayanz et al.

107 2002) and global (de Groot et al. 2006, Di Giuseppe et al. 2016) scale. These forecasting systems

108 are expected to improve as the performance of numerical weather prediction systems improve

109 (Bauer et al. 2015).

110 In general, the presence of systemic bias in modelled weather data remains an obstacle to

111 their adoption for fire weather monitoring where it is desirable that fire danger indices are

112 unbiased - that is, they are exact at spatial locations with observations. Therefore, while remote

113 sensing technologies and numerical weather models continue to improve, it is not yet clear that

114 their performance surpasses that of a dedicated ground station network combined with spatial

115 interpolation procedures. For example, Hanes et al. (in press) found that using precipitation

116 estimates from CaPA improved fire danger estimates only in regions with radar coverage.

117 The spatial interpolation of FWI component variables is challenging for several reasons.

118 First, due to resource and geographical constraints it is not always feasible to achieve a

119 sufficiently dense station density (ie. corresponding to the $40 \mathrm{~km}$ representation radius). Second, 
120 interpolating precipitation and wind speed is highly problematic, owing to their high variability,

121 particularly over complex terrain. It has previously been noted that summer precipitation is

122 difficult to interpolate due to the occurrence of convective (ie. short range) rain events

123 (Flannigan and Wotton 1989). Last, because each of the fuel moisture codes (FFMC, DMC, DC)

124 track fuel moisture using the previous days' values, there is a significant temporal

125 autocorrelation in their values. This is reflected in the time lag constants, which represent the

126 time required for each of the corresponding layers to lose approximately $63 \%$ (ie. $1-1 / e$ ) of their

127 moisture due to evapotranspiration (assuming dry equilibrium conditions). For FFMC, DMC and

128 DC these are respectively 2/3, 15 and 53 days (Lawson and Armitage 2008). This means that any

129 errors in estimation of moisture codes can propagate to estimations made for successive days.

130 In this paper, we revisit the issue of spatial interpolation of fire danger studied previously

131 (Flannigan and Wotton 1989; Flannigan et al. 1998; Tait and Zheng 2005; Sanabria et al. 2013).

132 In particular, we compare several common interpolation schemes for spatial mapping of fire

133 danger using the FWI System. Our study region is the Canadian province of Alberta, of which

$13457 \%$ is covered by the Boreal forest, an area that requires substantial fire management efforts.

135 The interpolation methods we consider include inverse distance weighting, two spline methods

136 and Kriging, a geostatistical method that offers several advantages over the other methods. We

137 also compare interpolation of the FWI input variables followed by calculation of FWI

138 components to directly interpolating the FWI components.

139 Data

140 Fire weather data were obtained for Alberta from the weather section of the Wildfire

141 Management Branch of Alberta Agriculture and Forestry. Specifically, daily values of primary

142 meteorological variables at 12:00 LST included 2m temperature, relative humidity, 10m wind 
143 speed and 24 hour accumulated precipitation as required by the FWI system. Secondary variables

144 included the calculated fuel moisture codes FFMC, DMC, and DC and the fire weather indices

145 FWI, BUI, ISI and DSR. The data were subset to include 20 years of fire weather data from

146 1995-2014. This relatively long data record should prevent some of the issues associated with

147 large inter-annual variability of weather patterns, particularly with regard to precipitation as

148 noted in previous studies that used shorter data records (Flannigan et al. 1998, Hanes et al., in 149 press).

150 Provincial fire weather stations are typically only operational during the fire season

151 (March to October in Alberta) with the majority of stations operational after May. Therefore, the

152 data were further subset to only include data for each year between the dates June 1st and August

153 31st (92 days) and to only include stations that were operational for the entire duration of this

154 period. Although the shorter time period restricts our study to summer fire weather, this

155 compromise allowed the inclusion of the greatest number of fire weather stations for each year of

156 analysis. Over the 20 year period the number of validation stations (total number of stations)

157 varied between a minimum of 68 (106) in 1995 to a maximum of 102 (149) in 2012.

158 Interpolation error is bounded only for points inside the convex hull of the interpolation 159 points (Powell 1994). Outside of the convex hull, estimations are considered extrapolation, and 160 are subject to greater uncertainty since their values may not be bounded. Therefore, in order to 161 reliably conduct the leave-one-out cross-validation procedure (see methods below) it was

162 necessary to interpolate fire weather to station locations within the convex hull formed by the

163 station network (see Figure 2). One of the interpolation procedures we tested, Akima spline

164 interpolation, often failed for points close to (but inside) the convex hull boundary so for this

165 method it was further necessary to only choose validation stations no closer than $75 \mathrm{~km}$ to the

166 Alberta border (see Figure 3). 


\section{Methods}

169 In this study, we compared the accuracy of four different interpolation techniques (and their

170 variations) for estimating fire weather variables. Specifically, we tested interpolation of the daily

171 meteorological variables that are inputs to the FWI System: Temperature (T), Relative Humidity

172 (RH), 24 hourly accumulated Precipitation (P) and Wind speed (WS); as well as FWI System

173 components: Fine Fuel Moisture Code (FFMC), Duff Moisture Code (DMC), Drought Code

174 (DC), Build-Up Index (BUI), Initial Spread Index (ISI), Fire Weather Index (FWI). Daily

175 Severity Rating (DSR), also an output of the FWI system, was not investigated because it is a

176 transformed value of the FWI output, and so does not represent any additional information

177 output of the FWI system. Because the inputs to the FWI System consist of the four

178 meteorological variables and the three previous days' moisture codes there are three different

179 procedures that can be used for estimating the FWI System components at new locations.

180 1. "Calculate first, interpolate later" (CI): Calculate FWI components at observed locations

181 and interpolate FWI components to unobserved locations.

182 2. "Interpolate first, calculate later" (IC): Interpolate meteorological variables and previous 183 day's moisture codes (denoted FFMC-PD, DMC-PD, DC-PD) to unobserved locations, 184 then calculate FWI components.

185 3. "Interpolate weather only first, calculate later" (IC2): Interpolate meteorological variables 186 to unobserved locations, calculate moisture codes at unobserved locations using previous 187 day's values (and interpolated meteorological variables) at those locations, and then 188 calculate FWI components. If previous day's moisture codes are not yet calculated at 189 unobserved locations, use interpolated values. 
190 In this paper, we test the performance of each of these procedures using a cross-validation

191 procedure. Previous works have concluded that the CI procedure provides the best results with

192 respect to root mean squared error (Flannigan et al. 1998, Tait and Zheng 2005); we revisit this

193 conclusion and show that the temporal autocorrelation in the moisture codes has a significant

194 influence on estimation error and bias for each procedure.

\section{Spatial interpolation Methods}

196 Currently the Alberta Wildfire Management Branch estimates fire weather between stations

197 using either the method of Inverse Distance Weighting (IDW) with weights given by the power

198 parameters $p=2$ or using the thin plate spline with smoothing (TPSS) (personal

199 communication). Therefore, these methods were included as baselines for comparison with the

200 other methods. The interpolation methods compared in this study were chosen because they are

201 commonly used methods, and they can be easily implemented in a number of open source and

202 propriety packages. Moreover, they are computationally fast making them suitable for daily

203 operational use. We outline the different methods used below.

204 Thin Plate Spline (TPS)

205 The Thin Plate Spline is a variational method that is a two dimensional generalization of cubic

206 splines (Duchon 1977; Wahba and Wendelberger 1980). In two dimensions the interpolating

207 surface is estimated by the function $f(r)=\sum_{i=1}^{K} \omega_{i} \varphi\left(\left\|r-r_{i}\right\|\right)$ for $K$ control points and where

$208 \varphi(r)=r^{2} \log (r)$. The function is found by minimizing

$$
\sum_{i=1}^{n}\left\|z\left(r_{i}\right)-f\left(r_{i}\right)\right\|^{2}+\lambda \iint\left[\left(\frac{\partial^{2} f}{\partial^{2} x}\right)^{2}+2\left(\frac{\partial^{2} f}{\partial x \partial y}\right)^{2}+\left(\frac{\partial^{2} f}{\partial^{2} y}\right)^{2}\right] d x d y
$$


210 where $z\left(r_{i}\right)$ is the observed value at $r_{i}$ and the integral represents the deformation energy of the

211 function. The parameter $\lambda$ determines the level of smoothing. $\lambda=0$ corresponds to no

212 smoothing so that the spline function passes through the data points (an unbiased estimator),

213 whereas $\lambda \neq 0$ can further minimize the deformation energy subject to a trade-off with

214 goodness-of-fit. We respectively denote these methods as TPSNS (thin plate spline with no

215 smoothing) or TPSS (thin plate spline with smoothing). Thin plate smoothing was implemented

216 using the fields package available for R (Nychka et al. 2015). By default, the smoothing

217 parameter is chosen automatically by a generalized cross validation procedure. The number and

218 location of knots is taken as the default, which corresponds to all unique locations.

219 Akima Spline (AS)

220 Akima spline (AS) (Akima 1978) interpolation is based on a triangular tessellation of all the data

221 points. A continuously differentiable piecewise function is then fit with each piece corresponding

222 to a cubic polynomial that passes through the vertices of each triangle. Specifically, within each

223 triangle the function is estimated by

$$
\hat{z}\left(r_{i}\right)=\sum_{j=0}^{3} \sum_{k=0}^{3-j} q_{j k} x_{i}^{j} y_{i}^{k}
$$

224 where the 10 coefficients $q_{j k}$ are found by solving the set of linear equations formed using the

225 set of points given by $r_{i}=\left(x_{i}, y_{i}\right)$ and its 9 closest neighbors. As this is a local approximator, it

226 can lead to a surface robust to outliers. This feature of the Akima spline motivates the inclusion

227 of this method in our study as it may lead to improved estimates for precipitation, a quantity that

228 can exhibit large spatial variability. Moreover, the Akima spline does not use additional

229 smoothing, and since it passes through all data points, is therefore an unbiased estimator. To

230 implement the Akima spline we used the akima package available for R (Akima et al. 2015). 
232 Inverse distance weighting (IDW) is a method that approximates a value at an unknown location

233 using a linear weighted average of the data at known points using the formula (Shepard 1968)

$$
\hat{z}\left(r_{i}\right)=\frac{\sum_{j=1}^{n} z\left(r_{j}\right) d_{i j}^{-p}}{\sum_{j=1}^{n} d_{i j}^{-p}}
$$

234 where $d_{i j}$ is the distance between the $i$ th and $j$ th points. Here we take $p=2$, which is the value

235 used by the Alberta wildfire management branch, which therefore provides a practical baseline

236 with which to compare the other methods. However, it should be noted that in general, $p$ can be

237 optimized by choosing the value which minimizes the absolute error. To implement IDW we

238 used the gstat package available for R (Pebesma 2004).

239 Kriging

240 Kriging is a geostatistical interpolation method (Cressie 2015; Wackernagel 2013) based on the

241 spatial covariance of the data. The method requires fitting a statistical model to the so-called

242 semivariogram, given by

$$
\gamma(\boldsymbol{h})=\frac{1}{2 N_{\boldsymbol{h}}} \sum_{i=1}^{N_{\boldsymbol{h}}}\left[z\left(r_{i}\right)-z\left(r_{i}+\boldsymbol{h}\right)\right]^{2}
$$

243 where $N_{\boldsymbol{h}}$ is the number of pairs of observation points separated by a distance $\boldsymbol{h}$. Using the fitted

244 model, the ordinary kriging (OK) estimator is given by the linear weighted average of known

245 values at neighboring points

$$
\hat{z}\left(r_{i}\right)=\sum_{j=1}^{n} \omega_{j} z\left(r_{j}\right)
$$

246 where the weights $\omega_{j}$ are determined by minimizing the error variance $\operatorname{var}\left[\hat{z}\left(r_{i}\right)-z\left(r_{i}\right)\right]$ with

247 the constraint that the expected value of the error is zero. OK is the most commonly used form of 
248 kriging, and is equivalent to spatial regression around a local mean estimated from the data.

249 Under the assumption that the data has a multivariate Gaussian distribution, OK provides the

250 best linear unbiased estimator (BLUE). In this paper, we employ OK as well as two variants:

251 Regression Kriging (RK) and Universal Kriging (UK). RK allows incorporation of auxiliary

252 variables by first fitting a regression model to the auxiliary variables and performing (ordinary)

253 kriging with the regression residuals. An obvious covariate for both meteorological and FWI

254 variables was elevation, available for each weather station in the Alberta fire weather database.

255 UK is a special case of RK where the spatial coordinates (longitude and latitude) are used as the

256 covariates. In this paper, we implemented OK, RK using elevation as a covariate (RKE) and UK.

257 Based on testing with several semivariogram models, we fit the semivariogram using the

258 spherical model for all variables considered here, which provided the best fit for the majority of

259 test cases. We also fit the semivariogram model separately for each day; we found fitting a single

260 semivariogram model for the entire season resulted in a poor fit due to the large temporal

261 variability in the weather data. To implement Kriging, we used the gstat package available for R

262 (Pebesma 2004).

\section{Transformations}

264 Data transformations can improve interpolation estimates for some variables, particularly those

265 that exhibit skewness in their distributions. The procedure is to transform the data using a

266 transformation function, perform the interpolation on the transformed data, and then back-

267 transform the interpolated values using the inverse of the transformation function. It should be

268 noted that this procedure can lead to a biased estimator for nonlinear transformations, for which a

269 bias correction term may be necessary (Cressie 2015). Both precipitation and wind speed are

270 non-negative quantities that exhibit skewness in their distributions (ie. non-normality), and their

271 interpolation may therefore be improved by transformation. It has been shown that the square 
272 root transform can reduce interpolation error for precipitation when using the thin plate spline

273 (Hutchinson 1998). In the current implementation of the Canadian Precipitation Analysis

274 (CaPA), that combines surface observations, numerical weather model predictions and radar

275 observations, the cube root transform has also been found to reduce estimation error (Fortin

276 2007). In the case of Kriging, if the data (or residuals for regression Kriging) are normally

277 distributed, the best unbiased predictor and best linear unbiased predictor are the same, so that

278 making the data approximately normal can similarly improve interpolation estimates. In this

279 paper, we considered three transformations (and corresponding back transformations) for

280 precipitation and wind speed, in addition to the default case of no transformation: (i) the square

281 root transform (SRT); (ii) the cube root transform (CBT); and (iii) a transformation based on the

282 natural $\operatorname{logarithm}(\ln )$ of the form $\ln (x+a)$ for a positive constant $a$, taken to be $a=0.1$. However,

283 we found the log transform consistently underperformed relative to the square root and cube root

284 transforms and therefore omit those results from the analysis. For interpolating relative

285 humidity, we tested a transformation to vapor pressure based on the Clausius-Clapeyron equation

286 (Satoh 2013); we found this method did not lead to any improvement over interpolating relative

287 humidity directly, so we omit these results also. For the kriging estimates, when the square root

288 or cube root transform was applied, we also tested the bias-corrected back-transformed values,

289 given respectively by (Gregoire et al. 2008)

$$
\begin{gathered}
\hat{z}(r)=\hat{y}(r)^{2}+\sigma_{y}^{2} \\
\hat{z}(r)=\hat{y}(r)^{3}+3 \hat{y}(r) \sigma_{y}^{2}
\end{gathered}
$$

290 where $\hat{y}(r)$ is the kriging estimate for the transformed variable and $\sigma_{y}^{2}$ is its variance. Note, it

291 was not possible to apply the bias correction procedure for either IDW, AS, TPSS or TPSNS

292 since none of the specific implementations used here provided the variance. 


\section{Cross-Validation}

295 In order to assess the performance of each interpolation method, a Leave-one-out cross-

296 validation (LOOCV) procedure was employed. For each year of analysis, the validation stations

297 were determined by the stations interior to the complex hull (Fig. 2). LOOCV proceeds by

298 looping through each validation station, removing observations of fire weather variables

299 corresponding to that station from the data, and interpolating the target variables to the location

300 of the removed station. The removed station data were then replaced and the procedure repeated

301 until all validation stations had been tested. The overall procedure was performed for each day

302 June $2^{\text {nd }}$ to August $31^{\text {st }}$. June $1^{\text {st }}$ was removed from the validation period as data from this date

303 was used to determine the values of the moisture codes, required as inputs to the FWI calculation

304 for June $2^{\text {nd }}$. Note that the interpolation procedure used the full set of available weather stations

305 comprising the validation stations as well as those on the complex hull boundary. In the special

306 case of Akima spline interpolation, the procedure was the same as above except that the

307 validation stations consisted only of those interior to a $75 \mathrm{~km}$ buffered region of the province

308 (Fig. 3).

309 To assess the performance of the various interpolation methods three continuous metrics

310 were considered: the mean absolute error (MAE), the mean rank absolute error (MRAE) and the

311 mean bias. They are defined respectively as follows:

$$
\begin{gathered}
\text { MAE }=\frac{1}{n} \sum_{i=1}^{n}\left|z_{m, i}-z_{o, i}\right| \\
\operatorname{MRAE}=\frac{1}{n} \sum_{i=1}^{n} \operatorname{rank}\left(\left\{\left|z_{m *, i}-z_{o, i}\right| \mid m * \leq M\right\}\right)
\end{gathered}
$$




$$
\text { mean bias }=\frac{1}{n} \sum_{i=1}^{n}\left(z_{m, i}-z_{o, i}\right)
$$

312 where $z_{o, i}$ and $z_{m, i}$ are the observed and predicted values at location $r_{i}, n$ is the total number of

313 observation-prediction value pairs and $m$ is a label that indexes the interpolation method. For the

314 MRAE, the rank is calculated between the set of methods $m *$ with $M$ being the total number of

315 methods being compared. In our case $n=n_{t} \times n_{s}$ where $n_{t}$ is the number of days in the validation

316 period (June $2-$ August $31^{\text {st }}$, ie. 91 days) and $n_{s}$ is the number of validation stations, which

317 varies by year. The MAE and mean bias represent the mean error across pairs of observations

318 and predicted values. MAE has the advantage over the root-mean-square error (RMSE) metric of

319 giving less weight to outliers. The MRAE is a non-parametric rank based measure.

To account for both spatial and temporal correlations in the data, as well as any non-

321 normality in the data, we employed a non-parametric vector block bootstrap method to determine

322 the $95 \%$ confidence intervals for each of the three metrics. This procedure involves repeated

323 resampling of the data (with replacement) using the stationary block bootstrap (SBB) (Politis and

324 Romano 1994) where each data element in the time series consisted of the vector of observations

325 (or predictions) at all validation stations corresponding to each day. Since each time point in the

326 resampled data includes observations at all spatial correlations, the spatial as well as temporal

327 correlation structure is preserved in the resampled data. The block length for each variable (see

328 Table 1) was determined by calculating the $95 \%$ confidence intervals for a range of block

329 lengths, and selecting the smallest block length above which the confidence intervals converged,

330 ie. were approximately constant. This ensured the minimum valid block length was chosen,

331 particularly important for DMC, DC and BUI, which exhibit the longest autocorrelation times. 
We first compared the seven interpolation methods (IDW, TPSS, TPSWS, AS, OK, UK,

336 RKE) for the meteorological variables that serve as inputs to the FWI System: ie. 24 hour

337 precipitation, wind speed, temperature and relative humidity. For both precipitation and wind

338 speed we additionally combined each method with either the square root transform (SRT) or

339 cube root transform (CRT). In the cases where kriging (OK, UK, RKE) was used with a

340 transformation we also tested the corrections to bias transformation as given in the methods

341 section (denoted SRTBC and CRTBC for SRT and CRT respectively). This resulted in a total of

34227 tested methods for these variables. The results are shown in Fig. 4. In the case of

343 precipitation, we find OK with the CRT transform give the lowest MAE, AS with CRT gives the

344 lowest MRAE and OK with SRT (bias corrected) gives the lowest mean bias. It should be noted

345 that for precipitation MAE values for each method did not differ appreciably and had relatively

346 large $95 \%$ confidence intervals for all tested methods. In contrast, the MRAE showed greater

347 differentiation between the methods compared with the confidence intervals. For wind speed the

348 RKE (no transformation) gives the lowest MAE and MRAE, whereas TPSS (no transformation)

349 gives the lowest mean bias. For both temperature and relative humidity, RKE (no

350 transformation) gives the best results with respect to all three metrics. Moreover, for temperature

351 it can be seen that the use of elevation as a covariate gives a significant improvement in the

352 interpolated estimates.

\section{Estimating FWI output variables}

354 To estimate the FWI System variables at new locations we test each of the three

355 procedures outlined in the methods section: The CI procedure corresponds to calculating the

356 FWI components at station locations and then interpolating the FWI output variables to new 
357 locations; The IC procedure corresponds to interpolating weather and the previous day's

358 moisture codes to unobserved locations and calculating FWI output variables; and the IC2

359 procedure proceeds similarly to IC except that the previous day's moisture codes are calculated

360 from the previous day's moisture codes at the unobserved locations. For IC we selected as inputs

361 the interpolated meteorological variables with the lowest MAE in each case. These were OK

362 with CRT for precipitation and RKE for wind speed, temperature and relative humidity. We also

363 tested two other combinations of interpolated weather variables, corresponding to either reduced

364 bias in precipitation or wind speed, to examine their influence on the FWI output variables.

365 Table 2 summarizes how each FWI input variable was selected for procedures IC and IC2.

366 Results for estimation of the moisture codes (FFMC, DMC, DC) at validation locations

367 using the three procedures is given in Fig. 5. We find for IC that calc-3 gives the lowest MAE for

368 FFMC, whereas calc-2 gives the lowest MAE for DMC and DC. IC2 (calc-4) performs relatively

369 poorly with respect to MAE and has the largest positive bias amongst all the estimation methods

370 for all moisture codes. In particular, for the DC, IC2 gives the largest MAE, MRAE and mean

371 bias. To further investigate the source of bias in the moisture code estimations we plot in Fig. 6

372 the daily mean bias (averaged over all validation stations) for procedures IC and IC2 for a typical

373 year, 2012 in this case. Here, calc-3 is omitted for clarity since it's results are identical to calc-1

374 for DMC and DC, which do not depend on wind speed, and calc-1 and calc-3 show almost

375 identical results for FFMC. For FFMC, calc-2 leads to the lowest bias of the calculated methods

376 in the first half of the study period, although there are some large negative deviations of bias in

377 the second half of the study period. For DMC and DC, calc-4 has a large and positive bias

378 compared with calc-1 and calc-2, which increases during the evaluation period. For DMC, calc-1

379 and calc-2 have negative biases that decrease during the evaluation period. For DC, calc-1 and

380 calc-2 do not change significantly during the evaluation period. 
382 locations using the three methods is given in Fig. 7. We find IC with interpolation of

383 precipitation using OK with SRT (bias corrected) (calc-2) leads to the lowest MAE for BUI, ISI

384 and FWI estimation. Calc-1, Calc-2 and Calc-3 perform similarly well with respect to MRAE for

385 ISI, BUI and FWI; whereas calc-4 (IC2) has smallest negative bias for ISI, AS the smallest

386 positive bias for ISI, calc-1/2 (IC) the smallest (negative) bias for BUI and TPSNS the smallest

387 (negative) bias for FWI. Similar to Fig. 6 we also plot the daily mean bias for the FWI output

388 indices (BUI, ISI and FWI) for 2012 in Fig. 8. For ISI there is no apparent trend in the mean bias

389 for any of the tested methods over the evaluation period. In contrast for both BUI and FWI, calc-

3904 exhibits a positive trend in mean bias, whereas calc- 1 and calc- 2 exhibit a negative trend in

391 mean bias.

\section{Autocorrelation of each variable}

To characterize the temporal autocorrelation of each variable we fit an exponential

394 function to the autocorrelation function (ACF) of each variable and defined the autocorrelation

395 time (ACT) as the time corresponding to a decay of the fitted ACF to a value of 0.1 (see Table

396 1). These values were then averaged over the 20 years of data used in the study. It is worth

397 noting that wind speed and precipitation show the shortest ACT values, whereas DMC, DC and

398 BUI show the longest ACT values. The long ACT times for DMC and DC are consistent with the

399 long (drying) lag times of those variables. Overall, in contrast to earlier studies (Flannigan et al.

400 1998; Tait and Zheng 2005) we achieved the best performance for estimating FWI variables with

401 long autocorrelation times (DMC, DC, BUI and FWI) when meteorological variables and

402 previous day's moisture codes were interpolated to unobserved locations and FWI was then

403 calculated at the unobserved locations using the interpolated values (ie. the IC procedure). 
406 estimation of each FWI output variable. The procedure is as follows. For each validation station

407 in the LOOCV procedure and each FWI input variable (T, RH, WS, P, FFMC-PD, DMC-PD,

408 DC-PD), we form the set of FWI input variables by taking the value of the selected variable from

409 the interpolated method which minimizes the MAE (given by RKE for T, RH, WS, FFMC-PD,

410 DMC-PD, DC-PD and OK-CRT for P) with the values of the remaining variables given by

411 observations. The set of input variables (interpolated and observed) is then used to calculate the

412 FWI output variables (FFMC, DMC, DC, ISI, BUI, FWI) and the corresponding MAE and mean

413 bias are calculated. The results are shown for the moisture codes (FFMC, DMC, DC) in Fig. 9

414 and for the fire behavior indexes (ISI, BUI, FWI) in Fig. 10.

415 For FFMC, DMC and DC the input variables that give the largest MAE are respectively

416 precipitation, DMC-PD and DC-PD. For FFMC, precipitation gives the largest (positive) bias;

417 for DMC, DMC-PD gives the largest (negative) bias; and for DC, DC-PD gives the largest

418 (positive) bias. For ISI, BUI and FWI the input variables that respectively give the largest MAE

419 are wind speed, DMC-PD and wind speed. For ISI, wind speed, relative humidity, precipitation

420 and FFMC-PD all contribute significantly to the overall bias; for BUI, DMC-PD gives the largest

421 (negative) bias; and for FWI, FFMC-PD and DMC-PD are the largest sources of (negative) bias.

\section{Discussion}

Improving the performance of spatial interpolation methods for the FWI System outputs

424 is crucial for more accurate spatial mapping of fire danger on the landscape, which is of obvious

425 benefit to fire management agencies. In this study, we compared several common interpolation

426 schemes for estimating FWI System outputs at unknown locations, and readdressed the question 
427 of whether it is best to first interpolate meteorological variables to unobserved locations and then

428 calculate the FWI System outputs or directly interpolate the FWI System outputs to unobserved

429 locations.

For the input meteorological variables, we found the geostatistical method Kriging

431 performed best with respect to mean absolute error. Specifically, temperature, relative humidity

432 and wind speed were interpolated with the lowest MAE using regression Kriging with elevation

433 as a covariate, whereas precipitation was interpolated with the lowest MAE using ordinary

434 Kriging with observations transformed using a cube root transform. It is worth noting that for

435 precipitation, although it is a local approximator, Akima spline interpolation did not perform

436 better than the Thin plate spline, indicating this feature may not have been exploited for the

437 spatial density of the data. Apart from reduced error, a benefit of using Kriging is that it is an

438 exact interpolator, meaning interpolated values are equal to observed values at sampled

439 locations; therefore, since FWI inputs are interpolated exactly at sampled locations, the

440 calculated FWI outputs are also exact at those locations.

441 Local orography (eg. elevation, slope aspect) can have a significant influence on climate

442 variables. There is, for example, a well-known inverse linear relationship between temperature

443 and elevation in the troposphere (ie. positive lapse rate). This relationship has previously been

444 exploited to improve interpolation of temperature (eg. Hudson and Wackernagel 1993, Daly et

445 al. 2002). Likewise, relative humidity, which varies with temperature and atmospheric pressure,

446 also has an implicit dependence on elevation. Although there is relatively little work on

447 interpolation of relative humidity, one study did find using cokriging with elevation as a

448 covariate led to the lowest estimation errors amongst several methods (Apaydin et al. 2004).

449 Elevation has also been previously used to improve interpolated values of wind speed (Luo et al.

450 2008). The positive correlation between precipitation and elevation in mountainous areas (Basist 
451 et al. 1994) has previously been exploited to improve interpolation estimates of precipitation (eg.

452 Goovaerts 2000, Daly et al. 2002). Including elevation as a covariate in our study led to the most

453 significant improvements for interpolation of temperature, with more modest improvements for

454 relative humidity and wind speed. Conversely, the three best performing methods - with similar

455 performance - for interpolating precipitation were ordinary kriging, universal kriging and

456 regression kriging with elevation (all using the cube root transform). The fact that regression

457 kriging with elevation did not outperform either ordinary nor universal kriging indicates may be

458 due the high spatial variability of precipitation combined and the supposition that the linear

459 relationship between precipitation and elevation is not as good as it is for the other variables (ie.

460 temperature, relative humidity and wind speed).

461 The performance of spatial interpolation of the moisture codes (FFMC, DMC, DC) and

462 fire behavior indexes (ISI, BUI, FWI) was significantly influenced by the procedure employed.

463 For FFMC we found both IC (calc-1, calc-2, calc-2) and IC2 (calc-4) perform well with respect

464 to MAE but that calc-2 gives a lower bias. For DMC and DC, IC (calc-1, calc-2, calc-3) and CI

465 (with RKE) perform well but IC2 (calc-4) performs relatively poorly. The lower bias achieved

466 with calc-2 for FFMC reflects the fact that the interpolated precipitation input has a lower bias.

467 For all moisture codes, calc-4 lead gave a large positive mean bias compared to the other

468 methods tested. This can be attributed to the propagation of estimation error from the previous

469 day's codes to the current day's codes, an effect which is larger for DMC and DC with large

470 autocorrelation times (Table 1).

471 To understand the performance of the estimation methods on the fire behavior indices

472 (ISI, BUI, and FWI) it is necessary to consider the variable dependence of the FWI System (Fig.

473 1). Overall, the IC and IC2 procedures outperformed CI for all three indexes. In particular, ISI,

474 which depends only on FFMC and wind speed, was best estimated by IC (calc-1, calc-2, calc-3) 
475 and IC2 (calc-4). Interestingly, calc-4 gives the lowest (negative) bias of the four methods for

476 BUI, but the largest (positive) bias for FFMC; whereas calc-2 gives the largest (negative) bias of

477 the four methods for BUI, but the smallest (positive) bias for FFMC. In contrast, BUI is best

478 estimated by IC (calc-1, calc-2, calc-3) or CI (RKE), but is poorly estimated by IC2 (calc-4); this

479 result reflects the performance of estimation methods for DMC and DC, which are inputs to BUI.

480 FWI, which combines ISI and BUI, is best estimated by IC (calc-1, calc-2, calc-3). The middling

481 performance of IC2 (calc-4) for FWI is likely due to a compromise between its good

482 performance for ISI and poor performance for BUI. In general, IC2 (calc-4) performed poorly for 483 any of the FWI System outputs with relatively large correlation times (Table 1); that is, for 484 DMC, DC, and BUI. In a spatial modeling context, we have investigated the differences between a "calculate

486 first, interpolate later" (CI) approach and a “interpolate first, calculate later” (IC) approach (Stein 487 et al. 1991). In general, unless the model is linear, these approaches may lead to different results 488 (Addiscott and Tuck 1996). Due to model nonlinearities, CI may not reproduce spatial 489 heterogeneities of the actual (unknown) model, whereas IC might lead to significant uncertainty 490 in the model inputs, depending on the number of input parameters to be interpolated and their 491 spatio-temporal characteristics. Thus, whether CI or IC yields better performance is likely to be a 492 trade-off between uncertainty in the model inputs (ie. interpolation error) and model nonlinearity. 493 For example, in a study of drought index estimation (Rhee and Carbone 2011) IC was found to 494 perform better than CI, whereas a study of areal interpolation of soil moisture (Stein et al. 1991)

495 found conversely that CI performed better than IC. In yet another study looking at the estimation 496 of reference evapotranspiration (Mardikis et al. 2005) found IC and CI procedures gave very 497 similar results. 
As the FWI System is a nonlinear empirical model, the IC and CI procedures will in

499 general yield different results, depending on the spatio-temporal characteristics of the FWI input

500 parameters. In the current study, we found that in most cases IC outperformed CI with respect to

501 both MAE and mean bias. In contrast, an earlier study on interpolation of the FWI System

502 indexes in north-western Ontario by Flannigan et al. (1998) found the CI procedure (ie.

503 interpolating the FWI indexes directly) performed better than the IC procedure. They attributed

504 this to the short-range nature of convective precipitation, which leads to relatively poor

505 estimation of this variable by interpolation. Tait and Zheng (2005) similarly suggested the CI

506 procedure should also be used for interpolation of fire weather in New Zealand. There are a

507 number of differences between the present study and that of Flannigan et al. (1998) that may

508 account for the contrasting results: (i) Flannigan et al. (1998) considered north-western Ontario,

509 whereas the present study considers Alberta. These regions have different climatologies and the

510 station networks also differ by location and distribution. In particular, the station density in

511 north-western Ontario was approximately one third of that considered in the present study; (ii)

512 Flannigan et al. (1998) did not test geostatistical methods, which in the present study have

513 further reduced interpolation error by inclusion of elevation or location as a covariate; (iii)

514 Flannigan et al. (1998) used 2 years of data with 6 validation stations, whereas the present study

515 uses a larger data set with 20 years of (summer) fire weather data with between 68 and 102

516 validation stations.

517 Since we found the IC procedure performed best overall, we also examined how

518 interpolation of each FWI input variable independently contributes to the output error and bias of

519 the FWI output variables (see Figs. 9 and 10). These results suggest which FWI input variable

520 interpolation estimates should be improved to give the largest reduction in estimation error (or

521 bias) for the FWI System outputs, thus providing guidance for possible future work. For 
522 example, improving estimates of precipitation, which leads to the largest MAE and mean bias for

523 FFMC, should lead to the largest improvement in FFMC. Likewise, improving interpolation of

524 DMC-PD and DC-PD should give the largest improvement in DMC and DC respectively. For

525 the fire behavior indexes, ISI and FWI may be best improved (with respect to MAE) by

526 improving interpolation of wind speed, and BUI should be best improved by improving

527 interpolation of DMC-PD.

\section{Conclusions}

529 In summary, the results of this study lead to two main recommendations for improving

530 interpolation of fire danger ratings based on the FWI System: (1) One should first interpolate the

531 FWI System input variables (temperature, relative humidity, wind speed, precipitation and

532 previous days' moisture codes) to unobserved locations and then calculate the FWI System

533 outputs; (2) regression kriging with elevation as a covariate should be used for estimating all

534 FWI System input variables, except for precipitation which may be better estimated using

535 ordinary kriging and the cube root transform.

536 There are two main directions any future work could take. First, an obvious strategy for

537 improving estimation of fire weather danger ratings is to improve interpolation of the FWI input

538 variables (particularly, those identified above) by either improving the methods used in this study

539 or by considering additional interpolation methods. In this study, we found the use of elevation

540 as a covariate improved interpolation for most of the considered variables. Incorporating

541 additional information may further improve interpolation estimates. For example, Wagner et al.

542 (2012) used wind and satellite remote sensing data as covariates for improved spatial

543 interpolation of precipitation; Jarvis and Stuart (2001) used topographic and land use variables to

544 improve interpolation of daily maximum and minimum temperatures; and Di Luzio et al (2008) 
545 considered additional topographic features for improving temperature and precipitation

546 estimates. Moreover, in addition to the interpolation methods considered in this study (ie. IDW,

547 splines and kriging) there are a number of other methods that are appropriate for environmental

548 variables including trend surface analysis (Tabios and Salas 1985), multivariate regression (Stahl

549 et al. 2006), conditional interpolation (Hewitson et al. 2005), objective analysis (Barnes 1964),

550 and machine learning algorithms (Li et al. 2011). Second, as an alternative to spatial

551 interpolation, gridded products for near-real-time monitoring of weather may be used. Such

552 systems are becoming more prevalent, including those based on satellite remote sensing (see

553 Thies and Bendex 2011 for a review), precipitation estimates that combine radar, observations

554 and numerical weather models (Mahfouf et al. 2007), and physics based wind models (Forthofer

555 et al. 2009). As these technologies continue to improve in the future, they are expected to be

556 incorporated into fire weather monitoring systems.

557 In Canada, the FWI System is an integral part of fire management operations at both a

558 daily and seasonal time scale. The use of the system is particularly important in Alberta where

559 total annual fire management expenditures have exceeded \$300million (CAD) in recent years

560 (Stocks and Martell 2016). Fire suppression costs can be associated with area burned, with 97\%

561 of the total area burned in Canada occurring for only $3 \%$ of the fires, corresponding to days with

562 extreme fire weather (Stocks et al. 2002). Accurate spatial mapping of fire weather is therefore

563 critical for fire management agencies to assess potential fire danger in regions where weather

564 stations are not located and to accordingly allocate resources for fire prevention or suppression,

565 or for emergency response.

566 


\section{Acknowledgements}

568 The authors would like to thank Alberta Environment and Parks for providing the fire weather

569 data. We also thank Peter Englefield for useful comments. 


\section{References}

572 Addiscott, T. M., and Tuck, G. 1996. Sensitivity Analysis for Regional-Scale Solute Transport

573 Modeling. In: D. L. Corwin, K. Loague, editors, Applications of GIS to the Modeling of Non-

574 Point Source Pollutants in the Vadose Zone, SSSA Spec. Publ. 48. SSSA, Madison, WI. p. 153-

575162.

576 Akima, H. 1978 "A method of bivariate interpolation and smooth surface fitting for irregularly

577 distributed data points." ACM Transactions on Mathematical Software (TOMS) 4.2: 148-159.

578 Akima, H., Gebhardt, A., Petzoldt, T., and Maechler M. 2015. akima: Interpolation of Irregularly

579 Spaced Data. R package version 0.5-12, https://CRAN.R-project.org/package=akima.

580 Apaydin, H., Sonmez, F. K., and Yildirim, Y. E. (2004). Spatial interpolation techniques for

581 climate data in the GAP region in Turkey. Climate Research, 28(1), 31-40.

582 Barnes, S. L. 1964. A technique for maximizing details in numerical weather map analysis.

583 Journal of Applied Meteorology, 3(4), 396-409.

584 Basist, A., Bell, G. D., and Meentemeyer, V. 1994. Statistical relationships between topography

585 and precipitation patterns. Journal of climate, 7(9), 1305-1315.

586 Bauer, P., Thorpe, A., and Brunet, G. 2015. The quiet revolution of numerical weather

587 prediction. Nature, 525(7567), 47-55.

588 Cressie, N. 2015. Statistics for spatial data. John Wiley \& Sons.

589 Daly, C., Gibson, W. P., Taylor, G. H., Johnson, G. L., and Pasteris, P. 2002. A knowledge-

590 based approach to the statistical mapping of climate. Climate research, 22(2), 99-113. 
591 Deeming, J. E., Burgan, R. E., and Cohen, J. D. 1977. The National Fire-Danger Rating System--

592 1978. USDA Forest Service General Technical Report INTUS (USA). no. 39.

593 de Groot, W.J., Goldammer, J.G., Keenan, T., Brady, M.A., Lynham, T.J., Justice, C.O., Csiszar,

594 I.A. and O'Loughlin, K., 2006. Developing a global early warning system for wildland fire.

595 Forest Ecology and Management, 234(1), p.S10.

596 Di Giuseppe, F., Pappenberger, F., Wetterhall, F., Krzeminski, B., Camia, A., Libertá, G., and 597 San Miguel, J. 2016. The potential predictability of fire danger provided by numerical weather 598 prediction. Journal of Applied Meteorology and Climatology, 55(11), 2469-2491.

599 Di Luzio, M., Johnson, G. L., Daly, C., Eischeid, J. K., and Arnold, J. G. 2008. Constructing 600 retrospective gridded daily precipitation and temperature datasets for the conterminous United 601 States. Journal of Applied Meteorology and Climatology, 47(2), 475-497.

602 Duchon, J., 1977. Splines minimizing rotation-invariant semi-norms in Sobolev spaces. In 603 Constructive theory of functions of several variables (pp. 85-100). Springer Berlin Heidelberg.

604 Englefield, P., Lee, B.S., and Suddaby, R.M. 2000. Spatial fire management system. Page Paper 605 \#489 in Proceedings from the ESRI International User Conference 2000, June 26-30, 2000, San 606 Diego, California. ESRI, San Diego, California, USA. 10 p.

607 Flannigan, M.D. and Harrington, J.B. 1988. A study of the relation of meteorological variables to 608 monthly provincial area burned by wildfire in Canada 1953-80. Journal of Applied Meteorology, $60927,441-452$.

610 Flannigan, M.D. and Wotton, B.M. 1989. A study of interpolation methods for forest fire danger 611 rating in Canada. Canadian Journal of Forest Research, 19, 1059-1066. 
612 Flannigan, M. D., Wotton, B. M., and Ziga, S. 1998. A study on the interpolation of fire danger

613 using radar precipitation estimates. International Journal of Wildland Fire, 8(4), 217-225.

614 Flannigan, M.D., Logan, K.A., Amiro, B.D., Skinner, W.R. and Stocks, B.J., 2005. Future area

615 burned in Canada. Climatic change, 72(1), pp.1-16.

616 Fortin, V., 2007: Analyse de précipitation CaPA: Proposition d'installation d'une passe

617 parallèle. Séminaire Recherche en Prévision Numérique, Dorval, QC, Canada, Environment

618 Canada, 65 pp.

619 Forthofer, J., Shannon, K., and Butler, B. 2009. Simulating diurnally driven slope winds with

620 WindNinja. In: Proceedings of 8th Symposium on Fire and Forest Meteorological Society; 2009

621 October 13-15; Kalispell, MT

622 Goovaerts, P. 2000. Geostatistical approaches for incorporating elevation into the spatial

623 interpolation of rainfall. Journal of hydrology, 228(1), 113-129.

624 Gregoire, T.G., Lin, Q.F., Boudreau, J. and Nelson, R., 2008. Regression estimation following

625 the square-root transformation of the response. Forest Science, 54(6), pp.597-606.

626 Haines, D.A. 1988. A lower atmosphere severity index for wildlife fires. National Emergency

627 Training Center.

628 Hanes, C.C., Jain, P., Flannigan, M.D., Fortin, V., and Roy, G. Evaluation of the Canadian

629 Precipitation Analysis (CaPA) to improve forest fire danger rating. International Journal of

630 Wildland Fire, 26(6), pp.509-522.

631 Hewitson, B.C. and Crane, R.G. 2005. "Gridded area-averaged daily precipitation via conditional 632 interpolation." Journal of Climate 18.1: 41-57. 
633 Hudson, G., and Wackernagel, H. 1994. Mapping temperature using kriging with external drift:

634 theory and an example from Scotland. International journal of Climatology, 14(1), 77-91.

635 Hutchinson, M. F. 1998. Interpolation of rainfall data with thin plate smoothing splines. Part I:

636 Two dimensional smoothing of data with short range correlation. Journal of Geographic

637 Information and Decision Analysis, 2(2), 139-151.

638 Jarvis, C. H., and Stuart, N. 2001. A comparison among strategies for interpolating maximum

639 and minimum daily air temperatures. Part I: The selection of "guiding" topographic and land

640 cover variables. Journal of Applied Meteorology, 40(6), 1060-1074.

641 Lawson, B.D., Armitage, O.B. 2008. Weather Guide for the Canadian Forest Fire Danger Rating

642 System. Natural Resources Canada, Canadian Forest Service, Northern Forestry Centre.

643 (Edmonton, Alberta, Canada)

644 Leblon, B. 2001. Forest wildfire hazard monitoring using remote sensing: A review. Remote

645 Sensing Reviews, 20(1), 1-43.

646 Lee, B. S., Alexander, M. E., Hawkes, B. C., Lynham, T. J., Stocks, B. J., and Englefield, P.

647 2002. Information systems in support of wildland fire management decision making in Canada.

648 Computers and Electronics in Agriculture, 37(1), 185-198.

649 Li, J., Heap, A. D., Potter, A., and Daniell, J. J. 2011. Application of machine learning methods

650 to spatial interpolation of environmental variables. Environmental Modelling \& Software,

$651 \quad 26(12), 1647-1659$.

$652 \mathrm{Li}$, J., and Heap, A. D. 2014. Spatial interpolation methods applied in the environmental

653 sciences: A review. Environmental Modelling \& Software, 53, 173-189. 
654 Li, Z.L., Tang, B.H., Wu, H., Ren, H., Yan, G., Wan, Z., Trigo, I.F. and Sobrino, J.A., 2013. 655 Satellite-derived land surface temperature: Current status and perspectives. Remote Sensing of 656 Environment, 131, pp.14-37.

657 Luo, W., Taylor, M. C., and Parker, S. R. 2008. A comparison of spatial interpolation methods to 658 estimate continuous wind speed surfaces using irregularly distributed data from England and 659 Wales. International journal of climatology, 28(7), 947-959.

660 McArthur A.G. 1967 Fire behaviour in eucalypt forest. Comm. Aust. For. Timb. Bur. Leaflet 107, $66125 \mathrm{p}$.

662 Mahfouf, J. F., Brasnett, B., and Gagnon, S. 2007. A Canadian precipitation analysis (CaPA) 663 project: Description and preliminary results. Atmosphere-Ocean, 45(1), 1-17.

664 Mardikis, M. G., Kalivas, D. P., and Kollias, V. J. 2005. Comparison of interpolation methods 665 for the prediction of reference evapotranspiration - an application in Greece. Water Resources 666 Management, 19(3), 251-278.

667 Nychka, D., Furrer, R., Paige, J. and Sain, S. 2015. "fields: Tools for spatial data.” doi: 668 10.5065/D6W957CT, R package version 8.10, www.image.ucar.edu/fields.

669 Pebesma, E.J., 2004. Multivariable geostatistics in S: the gstat package. Computers \& 670 Geosciences, 30: 683-691.

671 Politis, D. N., and Romano, J. P. 1994. The stationary bootstrap. Journal of the American

672 Statistical association, 89(428), 1303-1313.

673 Powell, M. J. D. 1994. The uniform convergence of thin plate spline interpolation in two 674 dimensions. Numerische Mathematik, 68(1), 107-128. 
675 Rhee, J. and Carbone, G. 2011: Estimating Drought Conditions for Regions with Limited 676 Precipitation Data. J. Appl. Meteor. Climatol., 50, 548-559

677 Sanabria, L. A., Qin, X., Li, J., Cechet, R. P., and Lucas, C. 2013. Spatial interpolation of 678 McArthur's Forest Fire Danger Index across Australia: Observational study. Environmental 679 modelling \& software, 50, 37-50.

680 San-Miguel-Ayanz, J., Barbosa, P., Schmuck, G., Liberta, G., and Schulte, E. 2002. Towards a 681 coherent forest fire information system in Europe: the European Forest Fire Information System 682 (EFFIS). Forest Fire Research and Wildland Fire safety. Millpress, Rotterdam, ISBN, 90-77017.

683 Satoh, M. 2013. Atmospheric circulation dynamics and general circulation models. Springer 684 Science \& Business Media.

685 Shepard, D. 1968. A two-dimensional interpolation function for irregularly-spaced data. In 686 Proceedings of the 1968 23rd ACM national conference (pp. 517-524). ACM.

687 Stahl, K., Moore, R. D., Floyer, J. A., Asplin, M. G., and McKendry, I. G. 2006. Comparison of 688 approaches for spatial interpolation of daily air temperature in a large region with complex 689 topography and highly variable station density. Agricultural and Forest Meteorology, 139(3), $690 \quad 224-236$.

691 Stein, A., Staritsky, I. G., Bouma, J., Van Eijnsbergen, A. C., and Bregt, A. K. 1991. Simulation 692 of moisture deficits and areal interpolation by universal cokriging. Water Resources Research, 693 27(8), 1963-1973.

694 Stephens, G. L., and Kummerow, C. D. 2007. The remote sensing of clouds and precipitation 695 from space: A review. Journal of the Atmospheric Sciences, 64(11), 3742-3765. 
696 Stocks, B.J., Mason, J.A., Todd, J.B., Bosch, E.M., Wotton, B.M., Amiro, B.D., Flannigan,

697 M.D., Hirsch, K.G., Logan, K.A., Martell, D.L. and Skinner, W.R., 2002. Large forest fires in

698 Canada, 1959-1997. Journal of Geophysical Research: Atmospheres, 107(D1).

699 Stocks, B. J., and Martell, D. L. 2016. Forest fire management expenditures in Canada: 1970-

700 2013. The Forestry Chronicle, 92(3), 298-306.

701 Tabios, G. Q., and Salas, J. D. 1985. A comparative analysis of techniques for spatial

702 interpolation of precipitation. JAWRA Journal of the American Water Resources Association,

$70321(3), 365-380$.

704 Tait, A., and Zheng, X. 2005. Final report: optimal mapping and interpretation of fire weather

705 information. NIWA Client Report No. WLG2005-1). Wellington: National Institute of Water and

706 Atmospheric Research.

707 Thies, B., and Bendix, J. 2011. Satellite based remote sensing of weather and climate: recent

708 achievements and future perspectives. Meteorological Applications, 18(3), 262-295.

709 Van Wagner, C.E. 1987. Development and structure of the Canadian Forest Fire Weather Index

710 System. Canadian Forestry Service, Headquarters, Ottawa. Forestry Technical Report 35. 35 p.

711 Wackernagel, H. 2013. Multivariate geostatistics: an introduction with applications. Springer

712 Science \& Business Media.

713 Wagner, P. D., Fiener, P., Wilken, F., Kumar, S., and Schneider, K. 2012. Comparison and

714 evaluation of spatial interpolation schemes for daily rainfall in data scarce regions. Journal of

715 Hydrology, 464, 388-400.

716 Wahba, G., and Wendelberger, J. 1980. Some new mathematical methods for variational 
717 objective analysis using splines and cross-validation. Monthly Weather Review 108: 1122-1145.

718 Wang, X., Thompson, D.K., Marshall, G.A., Tymstra, C., Carr, R. and Flannigan, M.D., 2015.

719 Increasing frequency of extreme fire weather in Canada with climate change. Climatic Change, 720 130(4), pp.573-586.

721 Williams, D.E. 1963. Forest fire danger manual. Can. Dept. For. Res. Branch, Publ. 1027, 722 Ottawa.

723

724

725 
727 Table 1: Autocorrelation times (ACT) and Stationary block bootstrap block lengths used for FWI input 728 and output variables.

\begin{tabular}{|l|l|l|l|l|l|l|l|l|l|l|}
\hline & T & RH & WS & P & FFMC & DMC & DC & ISI & BUI & FWI \\
\hline $\begin{array}{l}\text { ACT } \\
\text { (days) }\end{array}$ & 5.6 & 3.4 & 2.2 & 2.2 & 4.4 & 13.1 & 24.4 & 4.6 & 13.8 & 6.1 \\
\hline $\begin{array}{l}\text { SBB block } \\
\text { length (days) }\end{array}$ & 7 & 5 & 3 & 3 & 5 & 10 & 14 & 5 & 10 & 7 \\
\hline
\end{tabular}


731 Table 2: Estimation method used for each of the FWI System input variables for procedures IC and IC2. 732

\begin{tabular}{|c|c|c|c|c|c|c|c|}
\hline & $T$ & RH & WS & $\mathbf{P}$ & FFMC $_{t-1}$ & DMC $_{\mathrm{t}-1}$ & $D C_{t-1}$ \\
\hline $\begin{array}{l}\text { Calc-1 } \\
\text { (IC) }\end{array}$ & RKE & RKE & RKE & OK-CRT & RKE & RKE & RKE \\
\hline $\begin{array}{l}\text { Calc-2 } \\
\text { (IC) }\end{array}$ & RKE & RKE & RKE & OK-SRTBC & RKE & RKE & RKE \\
\hline $\begin{array}{l}\text { Calc-3 } \\
\text { (IC) }\end{array}$ & RKE & RKE & TPSS & OK-CRT & RKE & RKE & RKE \\
\hline $\begin{array}{l}\text { Calc-4 } \\
\text { (IC2) }\end{array}$ & RKE & RKE & RKE & OK-CRT & $\mathrm{FFMC}_{\mathrm{t}-2}$ & $\mathrm{DMC}_{\mathrm{t}-2}$ & $\mathrm{DC}_{\mathrm{t}-2}$ \\
\hline
\end{tabular}

733

734 


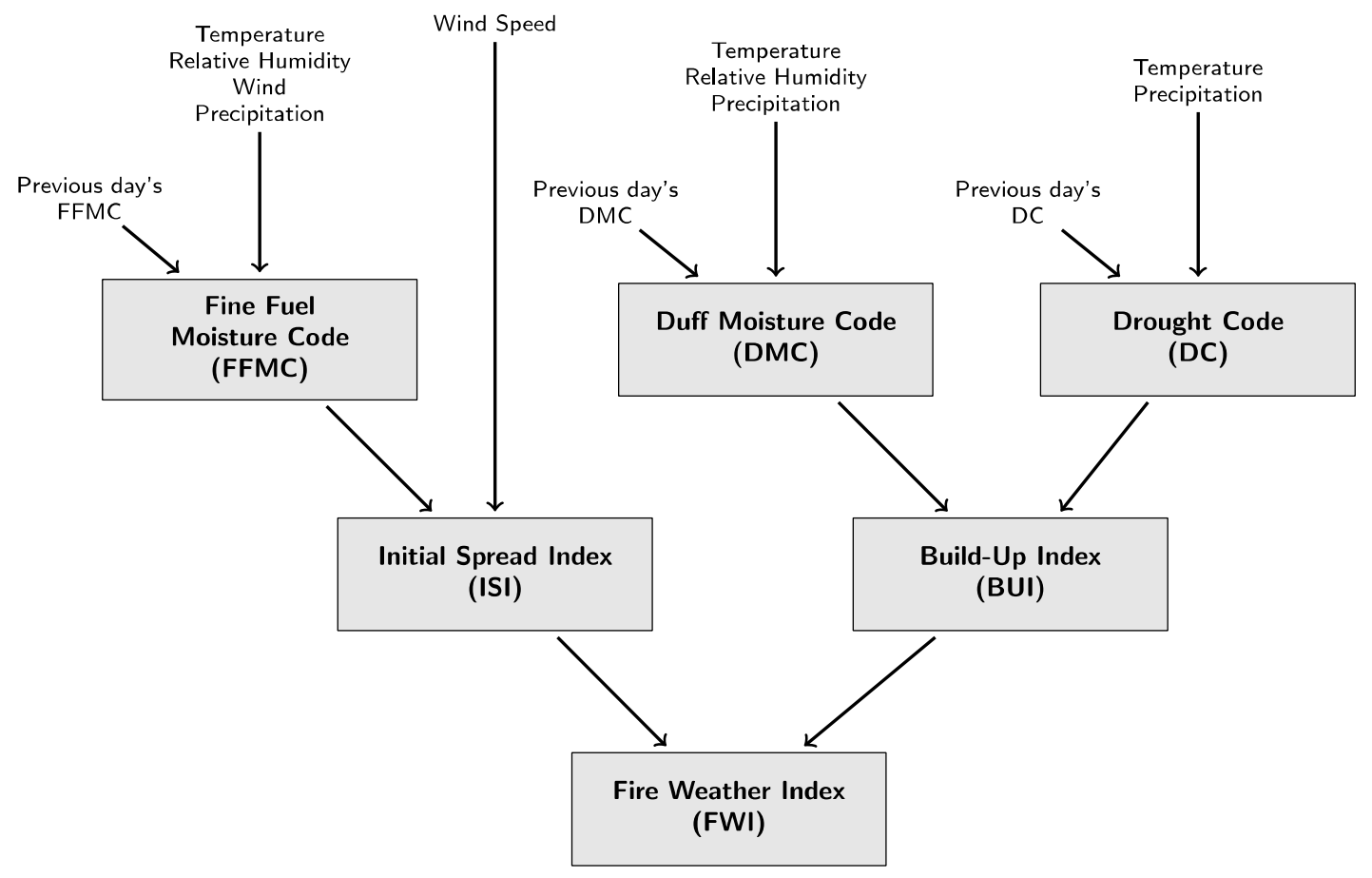

736 Figure 1: Variable dependence in the FWI system. 


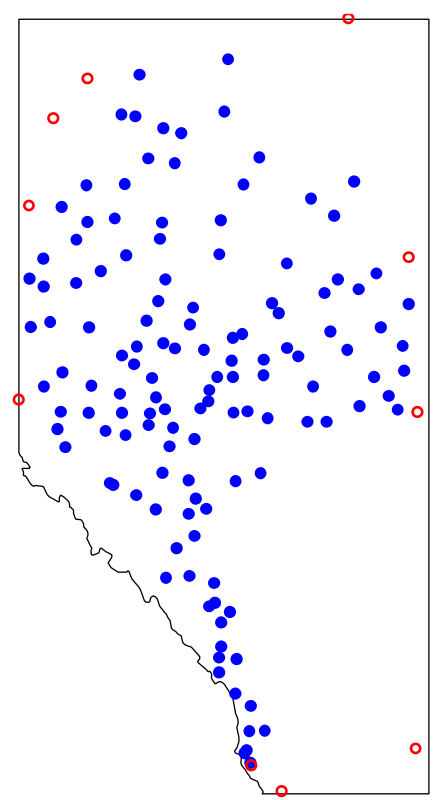

740 Figure 2: Operational fire weather stations for Alberta, June 1 - August 31 2014. Solid blue circles indicate stations

741 interior to the convex hull of all stations, whereas red hollow circles indicate stations comprising the convex hull. 
743

744

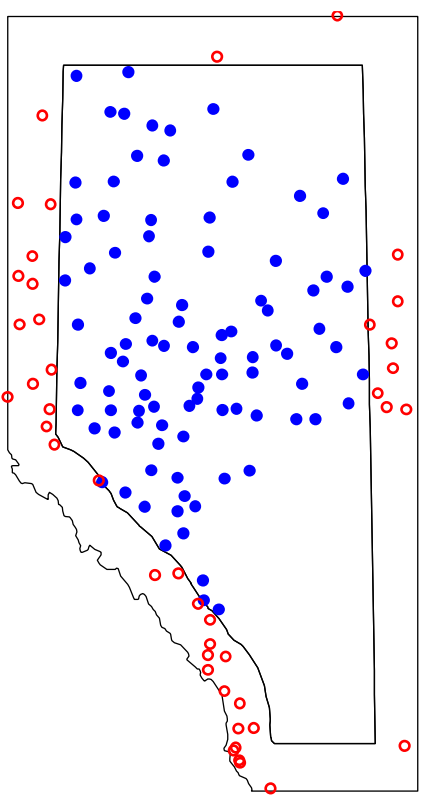

745

746 Figure 3: Operational fire weather stations for Alberta, June 1 - August 31 2014. Blue solid circles indicate stations

747 at least $75 \mathrm{~km}$ from the provincial border, whereas red hollow circles indicate stations located within $75 \mathrm{~km}$ of the

748 provincial border.

749 
(a) Precipitation
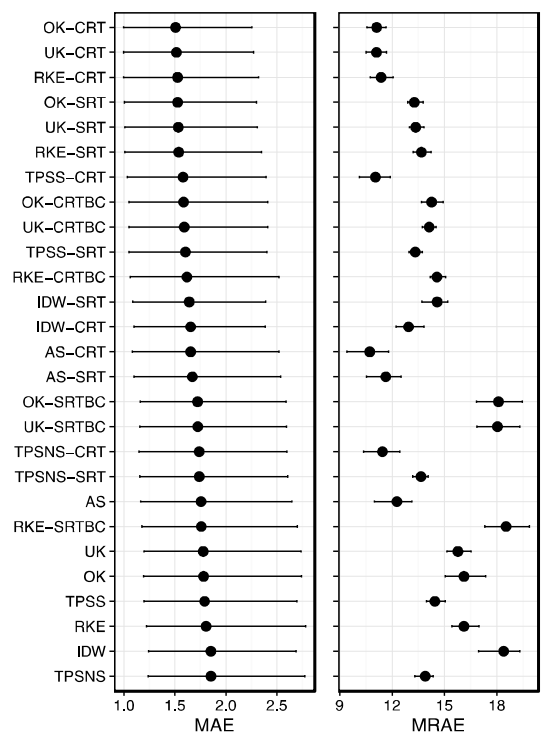

(c) Temperature

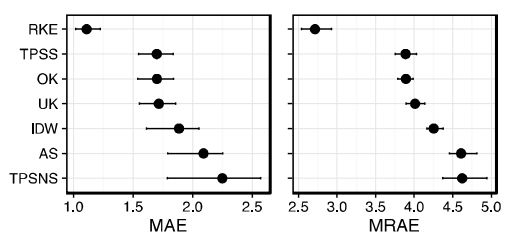

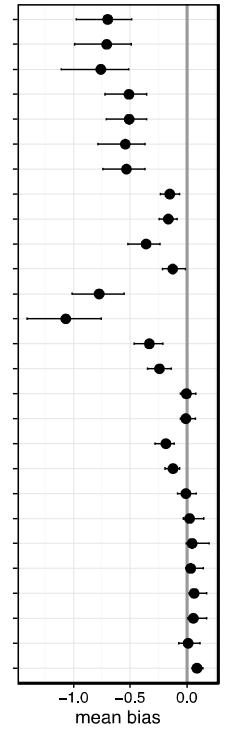

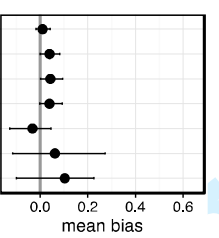

(b) Wind speed
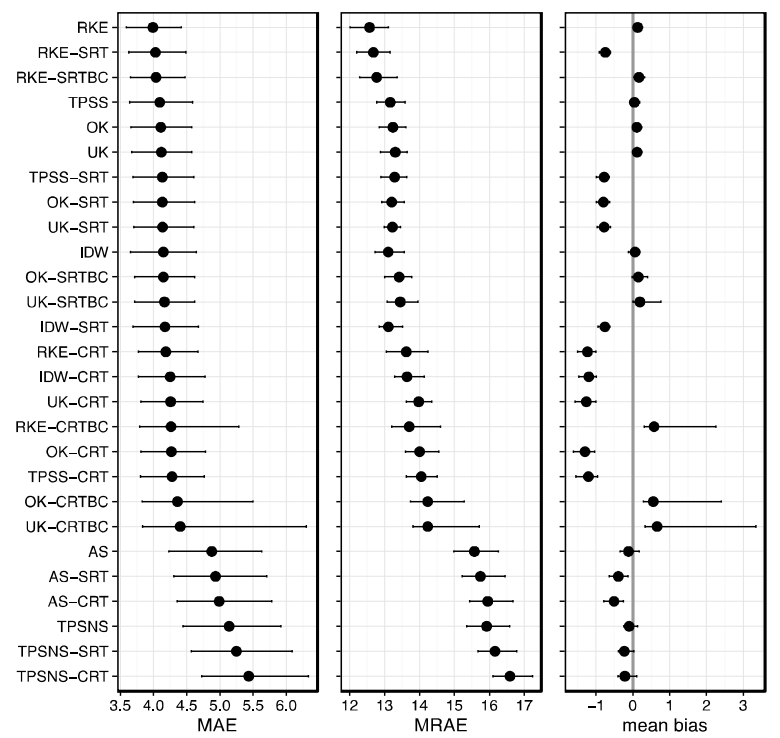

(d) Relative humidity
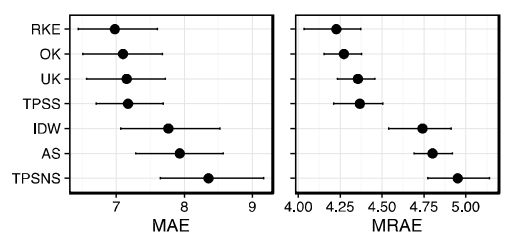

Figure 4: MAE, MRAE and mean bias for tested interpolation methods for FWI input meteorological variables.

752 Horizontal lines represent the 95\% percentile confidence intervals calculated using the bootstrap method outlined in

753 the main text.

754

755 
(a) FFMC
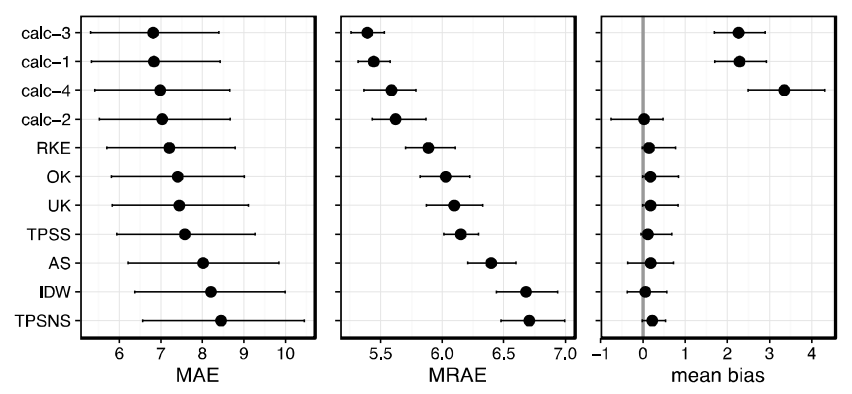

(b) DMC
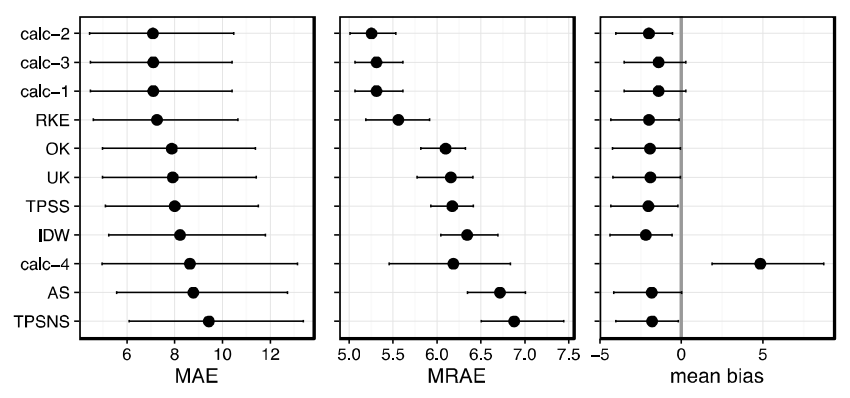

(c) DC
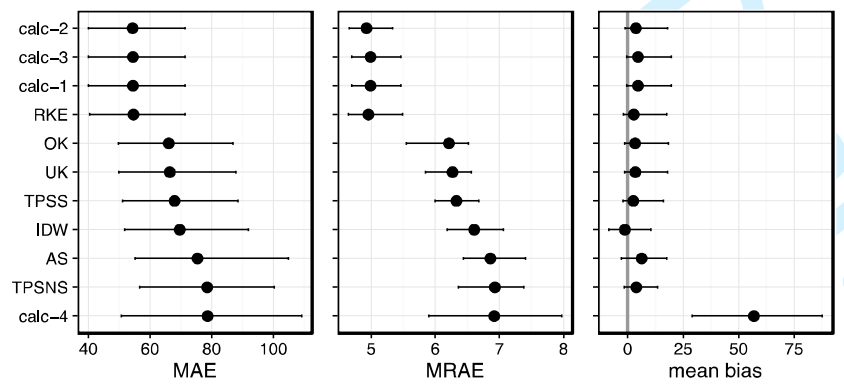

757 Figure 5: MAE, MRAE and mean bias for FWI System moisture codes estimated using different methods (see Table

7582 for definitions of calc-1, calc-2, calc-3 and calc-4 methods). Horizontal lines represent the 95\% percentile

759 confidence intervals calculated using the bootstrap method outlined in the main text. 

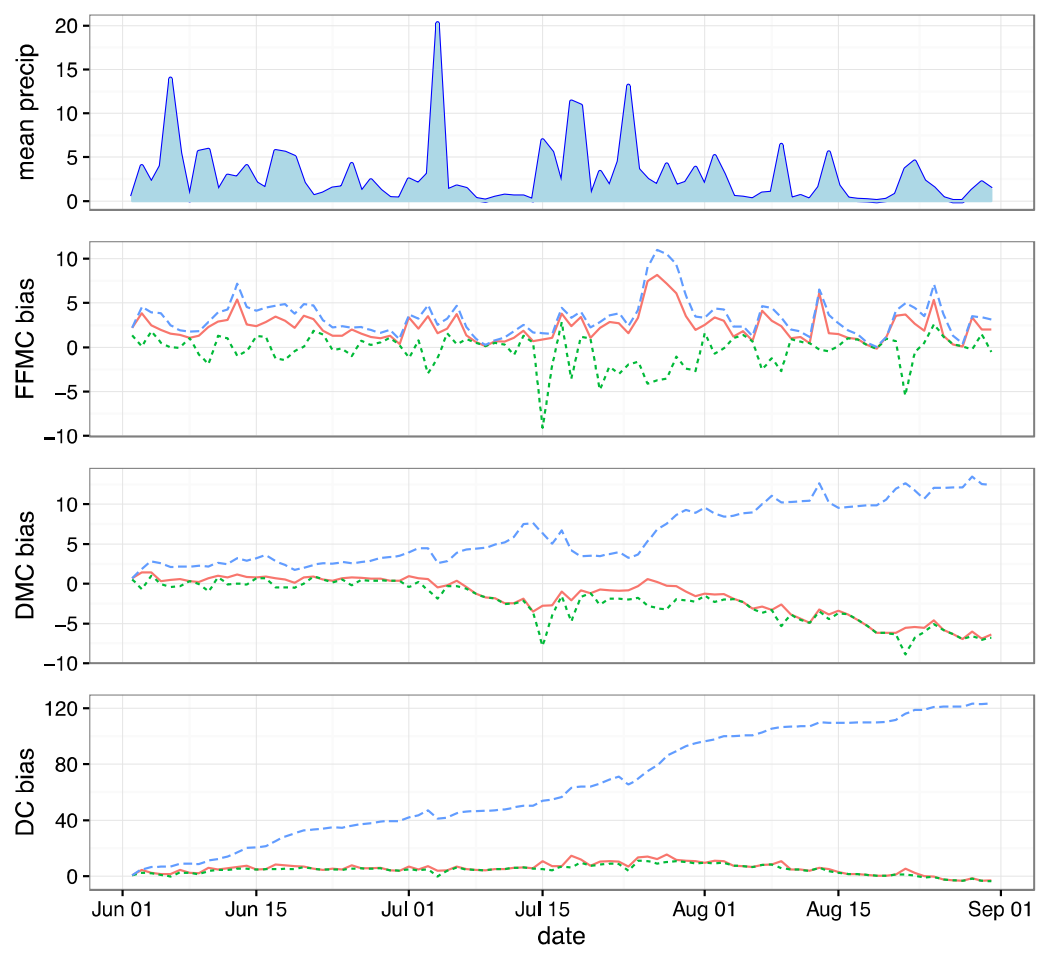

$\begin{array}{lll}\text { - calc-1 } & \text {-... calc-2 } & -- \text { calc-4 } \\ & & \end{array}$

761

762 Figure 6: Mean daily bias in moisture codes for different estimation methods. See main text for further details.

763 
(a) ISI
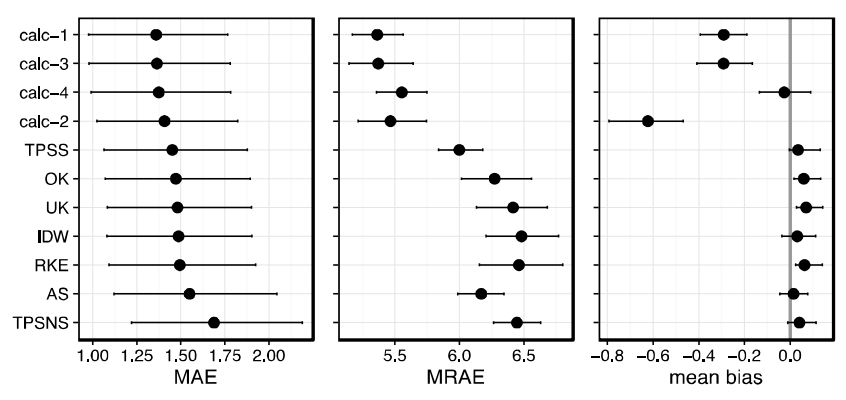

(b) BUI
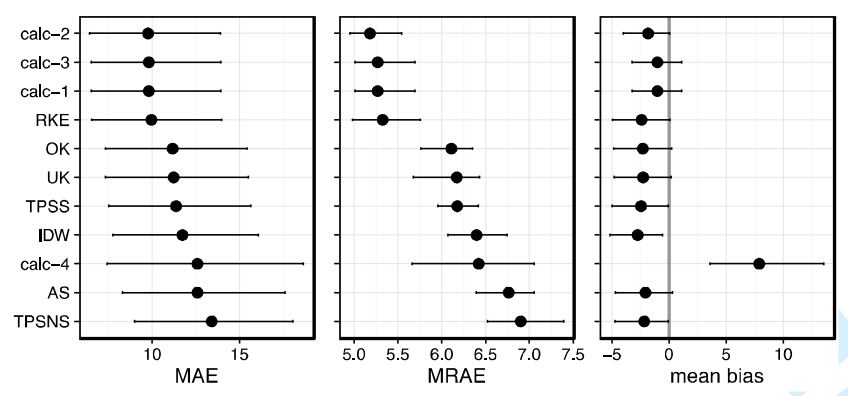

(c) FWI
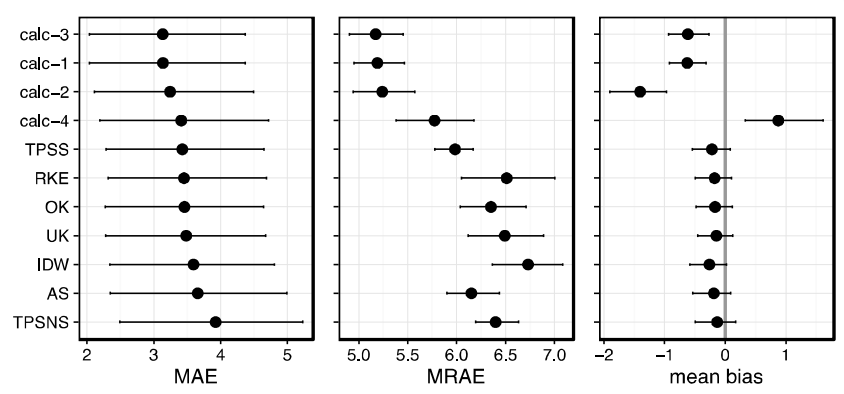

Figure 7: MAE, MRAE and mean bias for FWI System output variables estimated using different methods.

769 

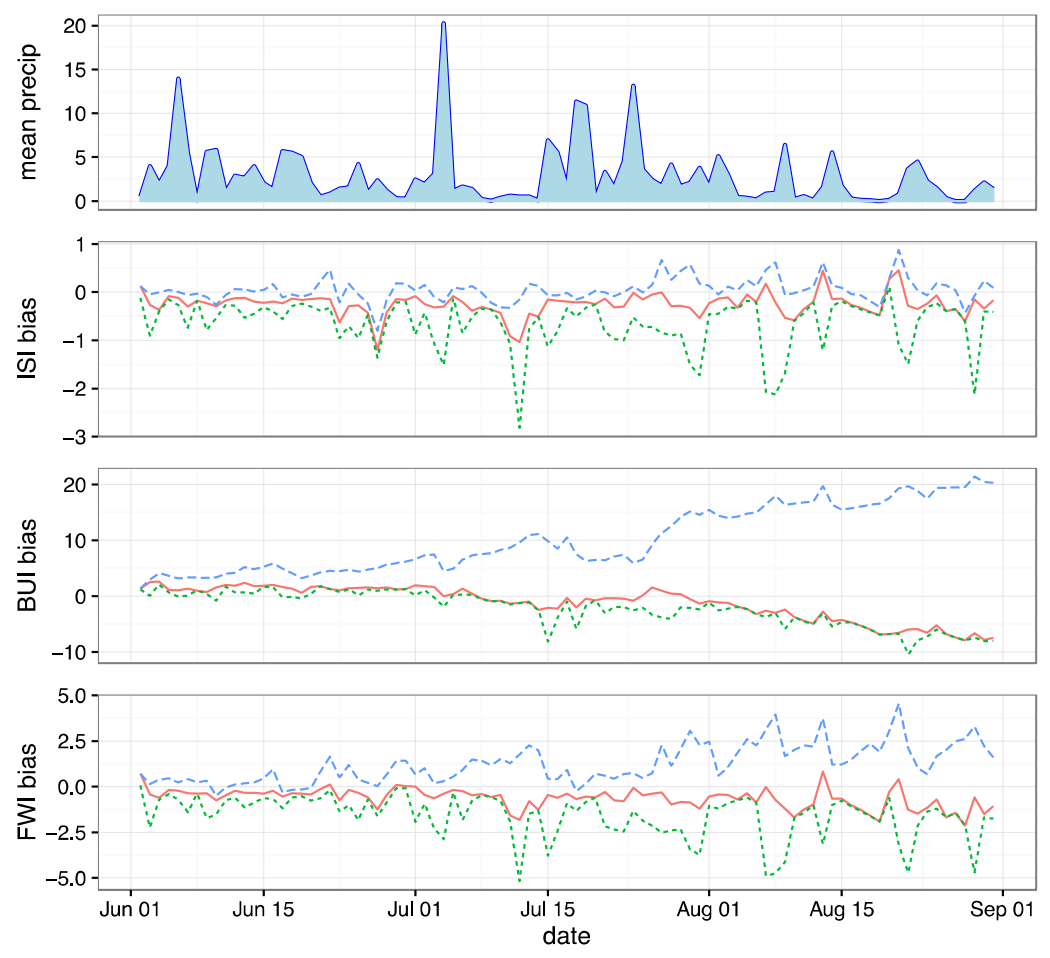

774 - calc-1 $\quad \cdots$ calc-2 $\quad--\cdot$ calc-4

775 Figure 8: Mean daily bias in FWI output indices for different estimation methods. See main text for 776 further details.

777

778

779

780 
(a) FFMC

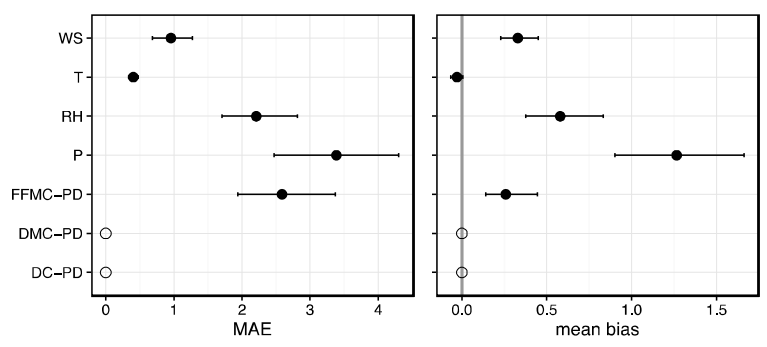

(b) DMC

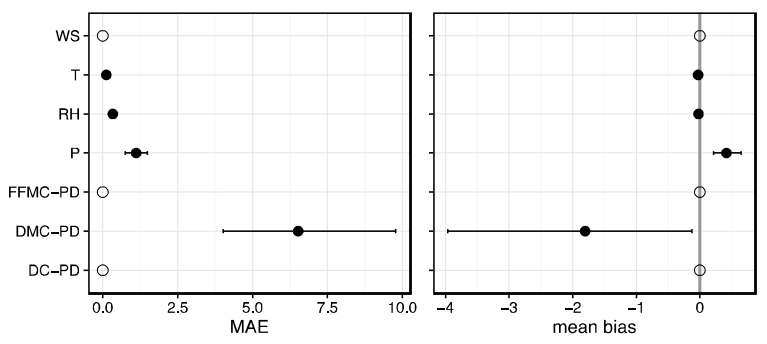

(c) DC
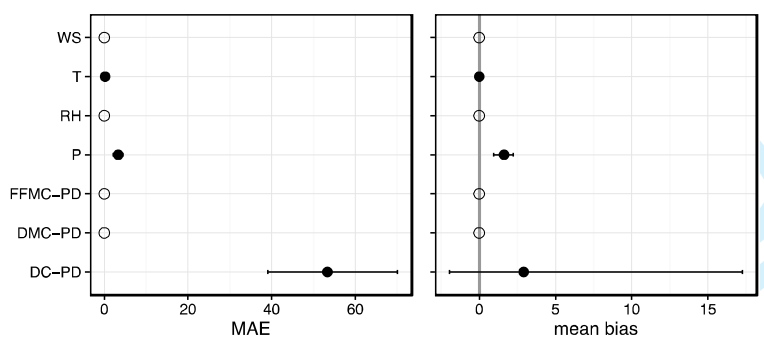

781

782 Figure 9: Variable importance for FWI moisture codes. Hollow circles indicate the moisture code has no 783 dependence on the variable.

784

785 


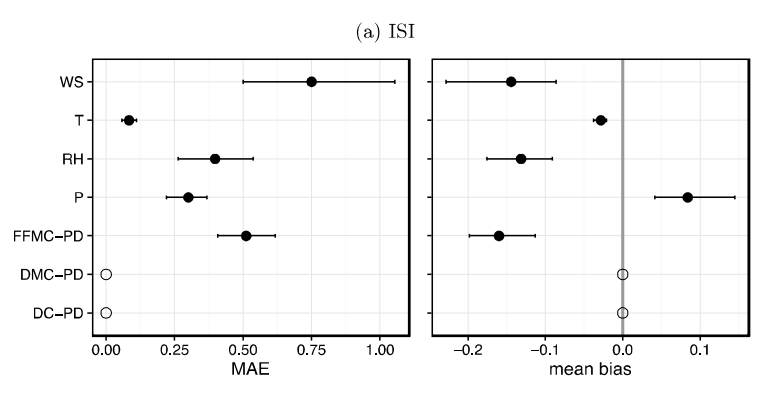

(b) BUI
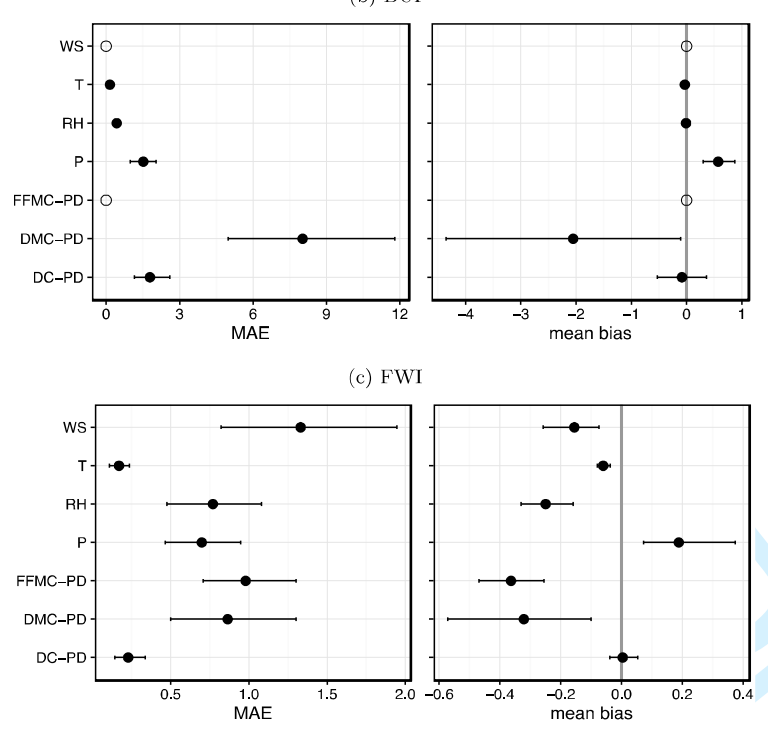

Figure 10: Variable importance for FWI fire behavior indexes. Hollow circles indicate the moisture code has no 789 dependence on the variable. 